\title{
Correlação das Variáveis Socioeconômicas e Ambientais com royalties Petrolíferos e CFEM Municipais
}

\author{
Liniker Fernandes da Silva ${ }^{1}$, Laércio Antônio Gonçalves Jacovine ${ }^{2}$, \\ Marcio Lopes da Silva ${ }^{2}$, Crismeire Isbaex ${ }^{2}$, Lyvia Julienne Sousa Rego ${ }^{2}$ \\ ${ }^{1}$ Centro de Ciências Agrárias, Ambientais e Biológicas, Universidade Federal do Recôncavo da Bahia - UFRB, \\ Cruz das Almas/BA, Brasil \\ ${ }^{2}$ Departamento de Engenharia Florestal, Universidade Federal de Viçosa - UFV, Viçosa/MG, Brasil
}

\begin{abstract}
RESUMO
O objetivo deste trabalho foi verificar a correlação entre variáveis ambientais, econômicas e sociais e os royalties arrecadados nos principais municípios produtores de petróleo e minério. Os dados discriminados por cidade, entre os anos de 2004 e 2010, foram a receita total das prefeituras, os investimentos em saneamento e gestão ambiental, o número de habitantes por município e o Índice FIRJAM de desenvolvimento municipal. Para verificar o relacionamento entre as variáveis utilizou-se a Análise de Componentes Principais. Os resultados mostraram que a maior arrecadação dos royalties, sejam do petróleo ou mineração, não tem como consequência direta maior desenvolvimento social ou ambiental. O que pode justificar tais resultados é a ausência de um dispositivo jurídico que condicione a aplicação dos recursos arrecadados às áreas em questão. Dessa forma, conclui-se que os possíveis impactos ambientais e sociais da exploração desses recursos não renováveis não estão sendo recompensados pelos royalties.
\end{abstract}

Palavras-chave: royalties do petróleo, CFEM, externalidades, desenvolvimento social, qualidade ambiental.

\section{Correlation of Socioeconomic and Environmental Factors with Municipal oil Royalties and CFEM}

\begin{abstract}
This study aimed to determine the correlation between environmental, economic and social variables with royalties collected in the main oil and mineral producing municipalities. The data used, disaggregated by city, between 2004 and 2010, were the municipalities' total revenue, investments in sanitation and environmental management, the number of inhabitants per municipality and Index FIRJAM municipal development. The relationship between the variables was evaluated by the Principal Component Analysis. The results showed that the largest collection of royalties, whether oil or mining, does not directly affect greater social or environmental development. Results can be explained by the lack of a legal framework that conditions the application of funds raised to the concerned areas. Thus, we conclude that the possible environmental and social impacts of exploitation of these non-renewable resources are not being rewarded for royalties.
\end{abstract}

Keywords: oil royalties, CFEM, externalities, social development, environmental quality. 


\section{INTRODUÇÃO}

Royaltyé uma palavra de origem inglesa e refere-se a remunerações de natureza diversa pagas pela concessão ou uso de direitos de autor ou proprietário de determinado bem. No Brasil, existem diferentes tipos de royalties implementados, dentre eles a Compensação Financeira pela Exploração de Recursos Minerais (CFEM) e os royalties sobre exploração do petróleo e gás natural.

A Compensação Financeira pela Exploração de Recursos Minerais (CFEM) é direito ao qual a união, os estados, o Distrito Federal e os municípios têm sobre os resultados da exploração dos recursos minerais em seu território (Brasil, 1988).

A CFEM é recolhida junto ao Departamento Nacional de Produção Mineral, sendo posteriormente destinada aos diferentes níveis do governo da seguinte forma: $23 \%$ aos estados de onde foi extraída a substância mineral, $65 \%$ aos municípios produtores, $10 \%$ ao DNPM (que deve destinar 2\% à proteção do meio ambiente nas regiões mineradoras, por meio do Ibama ou outro órgão federal competente) e $2 \%$ ao Fundo Nacional de Desenvolvimento Científico e Tecnológico (Brasil, 2000).

A alíquota da compensação incide sobre o faturamento líquido e varia de acordo com a atividade mineral. Potássio, minério de alumínio, manganês e sal-gema têm alíquota de 3\%. Ferro, fertilizantes e carvão têm alíquota de $2 \%$. Para o ouro, é de $1 \%$ e as pedras preciosas, carbonatos e metais nobres têm alíquota de 0,2\% (Brasil, 1990).

No tocante aos royalties do petróleo e gás natural, a compensação financeira devida aos estados, Distrito Federal e municípios corresponde a $10 \%$ sobre o valor bruto do recurso extraído do seu território ou localizados em instalações de embarque ou desembarque (Brasil, 2013).

A distribuição dos recursos provenientes de extração no continente é feita de forma que $70 \%$ é de direito dos estados produtores, $20 \%$, dos municípios produtores e $10 \%$ dos municípios em que se localizam as instalações marítimas de embarque ou desembarque do recurso (Brasil, 2013).

Já os recursos extraídos de plataformas marítimas são destinados de forma que $20 \%$ é de direito dos estados confrontantes, $3 \%$ dos municípios em que se localizam as instalações marítimas de embarque ou desembarque do recurso, $17 \%$ dos municípios confrontantes da atividade de exploração e $40 \%$ são destinados a dois fundos especiais, que destinam recursos a estados e municípios, e outros $20 \%$ são destinados a um fundo social (Brasil, 2013).

Os municípios fluminenses e espírito-santenses arrecadam juntos pouco mais de $70 \%$ dos recursos provenientes dos royalties do petróleo, de acordo com a Agência Nacional do Petróleo. Afinal, esses estados concentram a maior parte da produção de petróleo brasileira. Dessa forma, nesses estados ocorrem os maiores impactos sociais, ambientais e econômicos da atividade.

Os estudos acerca dos benefícios sociais e econômicos gerados pelos royalties do petróleo e da CFEM são inúmeros. Trabalhos como os de Neves et al. (2011) correlacionaram o volume de arrecadação de recursos dos royalties do petróleo com a qualidade das ações públicas, de forma a promover o desenvolvimento social e econômico dos municípios. Rodrigues \& Moreira (2013) e Ferreira (2013) avaliaram a gestão pública dos recursos provenientes da CFEM de forma a gerar benefícios sociais e econômicos para a população. A mesma disponibilidade de estudos não ocorre quando o objetivo é mensurar o impacto dos recursos provenientes dos royalties na questão ambiental.

Não existem na legislação regras que condicionem a aplicação de recursos dos royalties do petróleo ou da CFEM para melhoria das condições ambientais. Entretanto, os recursos minerais são de domínio da união (Brasil, 1988), de forma que os royalties devidos aos municípios não podem ser entendidos como uma participação nas receitas (Canotilho \& Leite, 2007 apud Thomé, 2009), visto que os mesmos não detêm a propriedade sobre tais recursos. Cientes da natureza compensatória dos royalties e somando a ausência de propriedade dos recursos por parte dos municípios, juridicamente pode-se interpretar que os royalties recebidos pelos municípios devem ser aplicados na recuperação do meio ambiente e no desenvolvimento social e econômico da região (Thomé, 2009; Ferreira, 1990).

Ciente da carência de estudos focados na gestão dos recursos provenientes dos royalties do petróleo e exploração mineral como forma de promover a gestão ambiental e sabendo das funções sociais, econômicas e ambientais da referida compensação, este trabalho teve como objetivo verificar a correlação entre as variáveis ambientais, econômicas e sociais com o volume de 
recursos arrecadados através dos royalties nos principais municípios produtores de petróleo e minério.

\section{MATERIAL E MÉTODOS}

\subsection{Escolha dos municípios}

Os municípios avaliados foram aqueles de maior arrecadação dos royalties do petróleo e da CFEM, de forma que o conjunto avaliado tenha representatividade no total de recursos recebidos pelos municípios brasileiros.

\subsection{Dados}

Para traduzir os aspectos econômicos dos municípios, utilizou-se a receita das prefeituras. As variáveis de cunho ambiental usadas foram os investimentos em saneamento e gestão ambiental. No tocante aos aspectos sociais, utilizou-se a população dos municípios e o Índice FIRJAM de Desenvolvimento Municipal (IFDM) para quantificar a qualidade dos aspectos sociais do município. Esse índice leva em consideração escolaridade, saúde, emprego e renda da população, sendo que quanto maior for o valor do índice melhor é a qualidade social do município.

Os dados relativos aos royalties do petróleo foram obtidos no site da Agência Nacional do Petróleo (ANP, 2015). Os valores da CFEM foram retirados do site do Departamento Nacional de Produção Mineral (DNPM, 2015). Os valores relativos à receita total, investimentos em saneamento e gestão ambiental de cada município estão disponíveis no site Compara Brasil, que é mantido pela frente nacional dos prefeitos. Já a população de cada município foi obtida no site do Instituto Brasileiro de Geografia e Estatística. O IFDM foi obtido junto à Federação das Indústrias do Rio de Janeiro (FIRJAN, 2015).

De posse das observações mencionadas, foi possível montar um banco de dados com todas as informações citadas, por município, compreendendo o período 2004 a 2010.

\subsection{Análise dos dados}

Tendo em vista o grande número de variáveis estudadas, utilizou-se uma análise multivariada denominada análise de componentes principais (PCA). Esta análise foi escolhida devido sua característica de entendimento da covariância entre as variáveis, de forma a indicar o relacionamento entre elas.

A análise de componentes principais reescreve os dados amostrados em um novo conjunto de eixos, denominados componentes principais, ortogonais entre si e cuja variância é decrescente da primeira para a última componente. Dessa forma, a primeira componente principal detém maior representatividade das informações que a segunda componente, e assim por diante. Tal procedimento reduz o número de informações que deve ser avaliado, facilitando o trabalho de interpretação.

$\mathrm{Na}$ análise de componentes principais foram utilizadas 183 observações, que compreendem os dados de royalties, população, investimentos em saneamento e gestão ambiental, índice IFDM, além da receita total de 26 prefeituras, entre 2004 e 2010. A análise foi realizada com o pacote Vegan do software R (Oksanen et al., 2011).

\section{RESULTADOS E DISCUSSÃO}

\subsection{Dados municipais avaliados}

Apenas 12 municípios, sendo que 11 deles são mineiros e 1 é paraense, concentram mais de $95 \%$ da arrecadação municipal da cfem, os royalties da mineração (Tabela 1). Desta forma, para a análise estatística, foram considerados esses municípios.

Com relação ao percentual arrecadado dos royalties do petróleo pelos municípios de cada estado, percebe-se que apenas os estados do Rio de Janeiro e Espírito Santo concentram mais de $70 \%$ dos valores arrecadados (Tabela 2), justificando o estudo de municípios desses estados.

Os municípios fluminenses de maior arrecadação no ano de 2013 estão discriminados na Tabela 3. Juntos, esses municípios tiveram em torno de $65 \%$ de toda a arrecadação dos municípios do estado com os royalties do petróleo. Destacam-se os municípios de Campos dos Goytacazes e Macaé, que juntos compreendem mais de 35\% da arrecadação.

Com relação ao Estado do Espírito Santo, apenas 7 municípios concentram quase $70 \%$ da arrecadação de royalties (Tabela 4), justificando o estudo dos mesmos. 
Tabela 1. Municípios de maior arrecadação da CFEM no ano de 2013.

Table 1. Cities' largest collection of the CFEM in 2013.

\begin{tabular}{|ccc|}
\hline Município & CFEM (2013) & \% do total \\
\hline Parauapebas - PA & $686.704 .191,14$ & 37,82 \\
\hline Nova Lima - MG & $233.906 .790,85$ & 12,88 \\
\hline Itabira - MG & $195.069 .791,18$ & 10,74 \\
\hline Mariana - MG & $139.924 .393,81$ & 7,71 \\
\hline São Gonçalo do Rio Abaixo - MG & $126.364 .766,56$ & 6,96 \\
\hline Itabirito - MG & $110.365 .845,83$ & 6,08 \\
\hline Brumadinho - MG & $77.742 .450,90$ & 4,28 \\
\hline Congonhas - MG & $67.979 .508,60$ & 3,74 \\
\hline Ouro Preto - MG & $40.466 .014,98$ & 2,23 \\
\hline Barão de Cocais - MG & $24.702 .656,39$ & 1,36 \\
\hline Santa Bárbara - MG & $16.025 .642,39$ & 0,88 \\
\hline Itatiaiuçu - MG & $15.908 .635,05$ & 0,88 \\
\hline Outros municípios & $80.690 .355,11$ & 4,44 \\
\hline Total & $\mathbf{1 . 8 1 5 . 8 5 1 . 0 4 2 , 7 9}$ & 100 \\
\hline
\end{tabular}

Fonte: DNPM (2015).

Tabela 2. Valor arrecadado dos royalties do petróleo pelos municípios, para os estados do Rio de Janeiro, Espírito Santo e os outros estados, em R $\$$ e $\%$, no ano de 2013.

Table 2. Collected value of oil royalties by city in the states of Rio de Janeiro, Espirito Santo and other states, in $\mathrm{R} \$$ and \%, in the year 2013.

\begin{tabular}{|ccc|}
\hline Estado & $\begin{array}{c}\text { Valor }(\mathbf{R} \$) \text { arrecadado pelos } \\
\text { municípios }\end{array}$ & $\begin{array}{c}\text { \% do total arrecadado por todos } \\
\text { municípios do Brasil }\end{array}$ \\
\hline Rio de Janeiro & $3.159 .202 .032,77$ & 56,59 \\
\hline Espírito Santo & $770.852 .880,34$ & 13,81 \\
\hline Outros estados & $1.652 .694 .208,82$ & 29,60 \\
\hline Total & $5.582 .749 .121,93$ & 100 \\
\hline
\end{tabular}

Fonte: ANP (2015).

Tabela 3. Valor arrecadado por município no estado do Rio de Janeiro, em R\$ e em \% do total arrecadado pelos municípios do estado, no ano de 2013.

Table 3. Value collected by city in the state of Rio de Janeiro, in R \$ and \%, of total municipalities in the state, in 2013.

\begin{tabular}{|ccc|}
\hline Município & $\begin{array}{c}\text { Royalties arrecadados (R\$) } \\
\text { Campos dos Goytacazes }\end{array}$ & $\begin{array}{c}\text { dotal arrecadado pelos } \\
\text { municípios no estado }\end{array}$ \\
\hline Macaé & $630.384 .833,22$ & 19,95 \\
\hline Cabo Frio & $466.531 .575,52$ & 14,77 \\
\hline Rio das Ostras & $186.552 .816,41$ & 5,91 \\
\hline São João da Barra & $186.682 .968,77$ & 5,91 \\
\hline Maricá & $107.493 .237,46$ & 3,4 \\
\hline Niterói & $102.961 .250,45$ & 3,26 \\
\hline Rio de Janeiro & $95.174 .814,06$ & 3,01 \\
\hline Quissamã & $89.958 .097,29$ & 2,85 \\
\hline Angra dos Reis & $85.684 .414,46$ & 2,71 \\
\hline Outros municípios & $72.117 .985,56$ & 2,28 \\
\hline Total & $1.135 .660 .039,57$ & 35,95 \\
\hline
\end{tabular}

Fonte: ANP (2015). 
Tabela 4. Valor arrecadado por município no estado do Espírito Santo, em R e em \% do total arrecadado pelos municípios do estado, no ano de 2013.

Table 4. Value collected by city in the state of Espirito Santo, in R $\$$ and \%, of total municipalities in the state, in 2013.

\begin{tabular}{|c|c|c|}
\hline Município & $\begin{array}{l}\text { Royalties arrecadados } \\
\text { (R\$) }\end{array}$ & $\begin{array}{l}\text { \% do total arrecadado pelos } \\
\text { municípios no estado }\end{array}$ \\
\hline Presidente Kennedy & $124.638 .790,03$ & 16,17 \\
\hline Linhares & $108.763 .835,03$ & 14,11 \\
\hline Itapemirim & $100.870 .193,62$ & 13,09 \\
\hline Marataízes & $73.123 .244,48$ & 9,49 \\
\hline São Mateus & $41.185 .328,17$ & 5,34 \\
\hline Aracruz & $38.483 .072,60$ & 4,99 \\
\hline Anchieta & $33.893 .493,25$ & 4,40 \\
\hline Outros municípios & $249.894 .923,16$ & 32,42 \\
\hline Total & $770.852 .880,34$ & 100 \\
\hline
\end{tabular}

Fonte: ANP (2015).

Para a realização das análises estatísticas, consideraram-se todos os municípios citados nas tabelas, exceto o município de Parauapebas. Essa opção é justificada pelo fato de o mesmo estar inserido em outra região geográfica brasileira, podendo assim sofrer influências sociais e políticas distintas das que ocorrem nos municípios da Região Sudeste.

\subsection{Análises estatísticas dos dados (análise dos componentes principais)}

As 3 primeiras componentes explicaram $94,2 \% \mathrm{da}$ variação total. Segundo o critério de Kaiser $(\bar{y}=0,803)$, deve-se utilizar as 3 primeiras componentes principais para a análise em questão visto que são as únicas que têm autovalores superiores à média de todos os autovalores (Figura 1).

Os valores dos loadings (Tabela 5) mostram que as variáveis Saneamento, Gestão e Receita municipal têm comportamento semelhante, visto que as mesmas apresentam valores semelhantes nas 3 componentes principais avaliadas. Tal comportamento pode ser visto de forma gráfica na Figura 2. A variável População se diferencia das variáveis anteriormente citadas somente na Componente Principal 2, dessa forma não se pode considerar a correlação da População com a Receita, Saneamento e Gestão Ambiental como alta. As variáveis Royalties e IFDM apresentam comportamento extremamente distinto em todas as componentes principais. Sendo assim, não se pode considerar a existência de correlação significante e positiva entre o recebimento de royalties e o desenvolvimento social, econômico e ambiental.

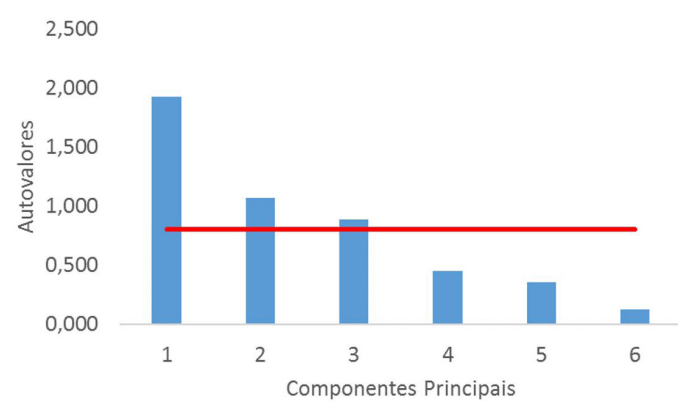

Figura 1. Variabilidade dos dados explicada por cada componente principal.

Figure 1. Variability of the data explained by each principal component.

A falta de relação direta entre as variáveis Royalties e Receita pode ser entendida de forma positiva uma vez que significa que os municípios avaliados, de forma geral, não têm as suas receitas totalmente dependentes da arrecadação de royalties. Afinal, é necessário lembrar que o petróleo e os minerais são recursos exauríveis e, dessa forma, uma dependência muito acentuada pode causar problemas no futuro.

A falta de relação entre os royalties e o IFDM pode ser entendida de forma que as atividades estudadas não geram, sozinhas, condições favoráveis ao crescimento da renda, geração de empregos, melhorias na educação e saúde. Esse resultado se soma aos obtidos por Neves et al. (2011), que verificaram a tendência entre as cidades fluminenses de maior arrecadação de royalties per capita terem menor IDH, enquanto as cidades de menor arrecadação per capita apresentavam IDH 
Tabela 5. Loadings das componentes principais.

Table 5. Loadings of the principal components.

\begin{tabular}{|cccccccc}
\hline & \multicolumn{7}{c}{ Componente principal } \\
\cline { 2 - 7 } Variável & $\mathbf{1}$ & $\mathbf{2}$ & $\mathbf{3}$ & $\mathbf{4}$ & $\mathbf{5}$ & $\mathbf{6}$ \\
\hline Royalties & 0,130 & 0,739 & $-0,622$ & $-0,096$ & $-0,194$ & 0,054 \\
\hline Saneamento & 0,481 & $-0,031$ & 0,052 & $-0,724$ & 0,452 & 0,191 \\
\hline Gestão & 0,476 & $-0,016$ & $-0,200$ & 0,638 & 0,567 & $-0,072$ \\
\hline Receita & 0,504 & $-0,147$ & 0,008 & $-0,104$ & $-0,402$ & $-0,743$ \\
\hline População & 0,491 & $-0,216$ & 0,037 & 0,178 & $-0,525$ & 0,635 \\
\hline IFDM & 0,171 & 0,620 & 0,754 & 0,132 & $-0,010$ & $-0,011$ \\
\hline Desvio padrão & 1,929 & 1,071 & 0,887 & 0,454 & 0,356 & 0,123 \\
\hline Var. acumulativa (\%) & 0,620 & 0,811 & 0,942 & 0,976 & 0,997 & 1 \\
\hline
\end{tabular}
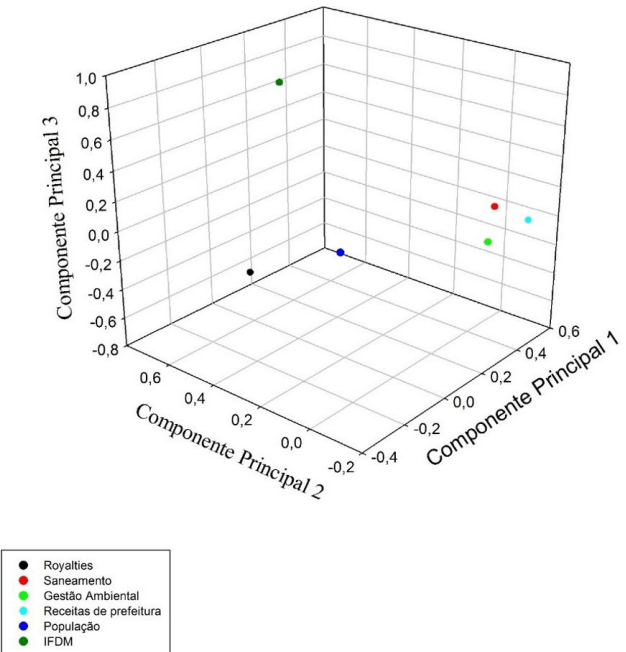

Figura 2. Gráfico do componente principal 1 versus componente principal 2 e componente principal 3.

Figure 2. Graph of principal component 1 versus principal component 2 and principal component 3.

superiores. Rodrigues \& Moreira (2013) verificaram que nos municípios mineiros de maior arrecadação per capita da CFEM, o montante arrecadado era inversamente proporcional ao Índice FIRJAM de Desenvolvimento Municipal.

A imprensa brasileira tem apontado, em diversas matérias, que os recursos provenientes dos royalties, sejam do petróleo ou da mineração, não estão sendo utilizados pelo poder público de forma a fomentar o desenvolvimento social e a manutenção das boas condições do meio ambiente. Entretanto, o enfoque estatístico, presente neste trabalho, permite comprovar cientificamente essas hipóteses e, ao mesmo tempo, ajuda a indicar possíveis alternativas para adequações na aplicação do dinheiro público.

A escassez de critérios legais que normatizassem a aplicação dos recursos provenientes tanto dos royalties do petróleo como da CFEM (Neves et al., 2011; Ferreira, 2013), no período avaliado, é um entrave à utilização dos mesmos com a finalidade de se melhorar as condições sociais e ambientais dos municípios. Além disso, segundo Silva (2000), a prestação de contas do uso desses recursos é falha. Assim, os órgãos públicos auditores e fiscalizadores, como o Ministério Público e os Tribunais de Contas, não podem questionar a aplicação dos recursos. Afinal, por mais que a aplicação não esteja sendo eficiente, ela não fere as leis.

Para minimizar esse problema, Euclydes (2013) propôs a vinculação de parte dos recursos obtidos através da CFEM a projetos de educação e proteção ambiental. Tal proposta também é válida para os recursos provenientes dos royalties do petróleo.

A associação entre as variáveis Saneamento e Gestão Ambiental e a variável população é de grande importância, pois significa que, de forma geral, o aumento da população é acompanhado de maiores investimentos na manutenção do equilíbrio ecológico. Nesse caso, essa associação não ocorreu.

A ausência de relação entre as variáveis Saneamento e Gestão Ambiental e a variável Royalties pode ser vista como preocupante. Afinal, maior arrecadação dos royalties ocorre em função da intensa atividade de extração petrolífera e mineradora. Essas atividades são altamente impactantes (Considine, 2014), gerando danos ambientais e influenciando outras atividades econômicas em função desse dano. Esse é o conceito de externalidade (Varian, 2010). Pode-se dizer que, do 
ponto de vista social e ambiental, as externalidades não estão sendo internalizadas, visto que nos municípios de maior faturamento dos royalties e CFEM o IFDM não apresenta a tendência clara de ser maior, bem como os investimentos em saneamento e gestão ambiental.

Os municípios estudados se localizam em áreas de Mata Atlântica. A mesma é um bioma com alta biodiversidade, com grande quantidade de espécies endêmicas e extremamente degradado, sendo por isso considerada um hotspot. Esse fato realça a importância de se investir na qualidade do meio ambiente nesses municípios.

A Figura 3 mostra a distribuição dos scores ao longo das componentes principais 1, 2 e 3 . Observa-se que os municípios de Minas Gerais, Espírito Santo e a maioria dos municípios fluminenses apresentam comportamento semelhante em todas as componentes. Quanto maior a arrecadação dos royalties, ou CFEM nos casos mineiros, maiores os investimentos em gestão, saneamento e IFDM.

Campos dos Goytacazes e Macaé (que são os de maior arrecadação de royalties) têm comportamento distinto. Mesmo apresentando valores positivos para a componente principal 2, têm valores negativos ao longo da componente principal 3. Lembrando que a
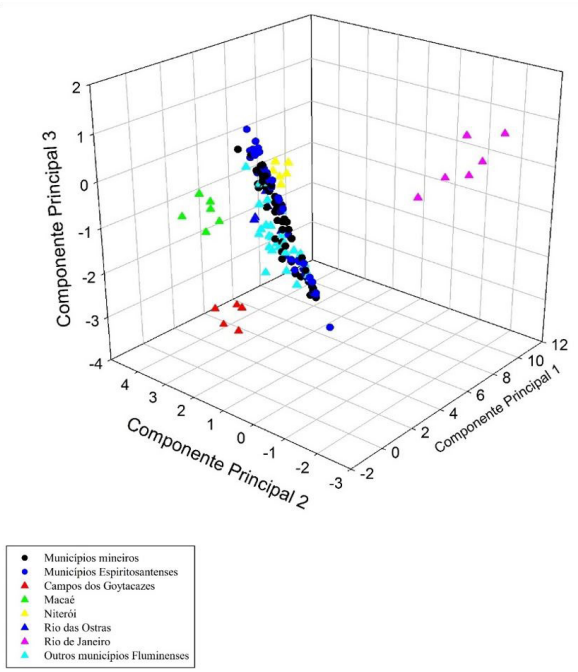

Figura 3. Scores dos municípios estudados, entre 2004 e 2010, na componente principal 1 versus as componentes principais 2 e 3 .

Figure 3. Scores of studied cities, between 2004 and 2010 , on the principal component 1 versus the principal components 2 and 3 . componente principal 2 explica principalmente a variável royalties e a componente 3 representa basicamente o IFDM, entende-se que para esses municípios não está havendo desenvolvimento social atrelado à arrecadação dos royalties.

Esses resultados concordam com o descrito por Seabra et al. (2015). Os mesmos autores concluíram que, na Bacia de Campos, o desenvolvimento econômico gerado pela atividade petrolífera não trouxe desenvolvimento social. Um dos grandes motivos para isso é, de acordo com os autores, a má aplicação dos royalties do petróleo feita pelas prefeituras desses municípios, entrando em conflito com a natureza compensatória do mesmo.

O Rio de Janeiro apresenta comportamento inverso às cidades mencionadas acima, pois apresenta valores negativos para a componente principal 2 e positivos para a componente principal 1. Nesse caso pode haver duas interpretações. Na primeira, o município utiliza seus royalties de forma a melhorar as condições sociais e, na segunda, o desenvolvimento do município é atrelado à sua receita total, representada pela componente 1 .

\section{CONCLUSÕES}

A arrecadação de royalties, seja do petróleo ou da mineração, não tem como consequência direta maior desenvolvimento social. Na verdade, casos com arrecadação mais alta tiveram resultados totalmente opostos. Dessa forma, a natureza compensatória dos royalties não vem sendo cumprida.

No contexto da economia ambiental, entende-se que as externalidades ambientais geradas pelas atividades mineradora e petrolífera não estão sendo internalizadas. Afinal, mesmo com o pagamento dos royalties, os gastos com gestão ambiental e saneamento não sofrem variação. Isso posto, a internalização não ocorre por causa da governança pública, que aplica de forma ineficiente os recursos arrecadados com os royalties.

\section{AGRADECIMENTOS}

Os autores agradecem à CAPES e ao CNPQ.

\section{STATUS DA SUBMISSÃO}

Recebido: 30 mar., 2015

Aceito: 27 jul., 2016 
AUTOR(ES) PARA CORRESPONDÊNCIA

\section{Liniker Fernandes da Silva}

Centro de Ciências Agrárias, Ambientais e Biológicas, Universidade Federal do Recôncavo da Bahia - UFRB, Rua Rui Barbosa, 710, Centro, CEP 44380-000, Cruz das Almas, BA, Brasil e-mail: liniker@ufrb.edu.br

\section{REFERENNCIAS}

Agência Nacional do Petróleo - ANP [online]. Brasília: ANP; 2015 [citado em 2015 mar 30]. Disponível em: http://www.anp.gov.br/?pg $=72182 \& \mathrm{~m}=59119 \& \mathrm{t} 1=\& \mathrm{t} 2=5$ 9119\&t3=\&t4=\&ar=0\&ps $=1 \&$ cachebust $=1409700061380$

Brasil. Constituição da República Federativa do Brasil. Diário Oficial da República Federativa do Brasil, Brasília, DF (1988 out. 05).

Brasil. Lei $n^{\circ}$ 8.001, de 13 de março de 1990. Define os percentuais da distribuição da compensação financeira de que trata a Lei no 7.990, de 28 de dezembro de 1989, e dá outras providências. Diário Oficial da República Federativa do Brasil, Brasília, DF (1990 mar. 14).

Brasil. Lei $n^{\circ} 9993$, de 24 de julho de 2000. Destina recursos da compensação financeira pela utilização de recursos hídricos para fins de geração de energia elétrica e pela exploração de recursos minerais para o setor de ciência e tecnologia. Diário Oficial da República Federativa do Brasil, Brasília, DF (2000 jul. 25).

Brasil. Lei $n^{\circ} 12.734$, de 30 de novembro de 2012. Modifica as Leis $n^{\circ}$ 9.478, de 6 de agosto de 1997, e $n^{\circ} 12.351$, de 22 de dezembro de 2010, para determinar novas regras de distribuição entre os entes da Federação dos royalties e da participação especial devidos em função da exploração de petróleo, gás natural e outros hidrocarbonetos fluidos, e para aprimorar o marco regulatório sobre a exploração desses recursos no regime de partilha. Diário Oficial da República Federativa do Brasil, Brasília, DF (2013 nov. 14).

Considine TJ. Economic and environmental impacts of oil and gas development offshore the Delmarva, Carolinas, and Georgia. 1. ed. Laramie: Interstate Policy Alliance; 2014 [citado em 2015 mar 30]. Disponível em: https:// www.heartland.org/policy-documents/economic-andenvironmental-impacts-oil-and-gas-developmentoffshore-delmarva-caroli
Departamento Nacional de Produção Mineral - DNPM [online]. Brasília: DNPM; 2015 [citado em 2015 mar 30]. Disponível em: http://www.dnpm.gov.br/

Euclydes ACP. Contradições da política ambiental por meio de incentivos financeiros: os casos do ICMS ecológico e da CFEM nos municípios do Quadrilátero Ferrífero (Minas Gerais, Brasil). Revista Árvore 2013; 37(6): 1083-1092. http://dx.doi.org/10.1590/S0100-67622013000600010.

Federação das Indústrias do Rio de Janeiro - FIRJAN. Índice FIRJAN de desenvolvimento municipal [online]. Rio de Janeiro: FIRJAN; 2015 [citado em 2015 mar 30]. Disponível em: http://www.firjan.org.br/ifdm/

Ferreira MG Fo. Comentários à Constituição brasileira de 1988. 1. ed. São Paulo: Saraiva; 1990.

Ferreira TR. Royalties minerais e capacidade de gestão em governos locais: um estudo em municípios mineradores de Minas Gerais [dissertação]. São Paulo: Fundação Getúlio Vargas; 2013.

Neves JA, Oliveira MS, Batista VC. Aplicação dos royalties do petróleo: uma proposta de avaliação qualitativa da eficácia das ações de governo. In: Anais do VIII Simpósio de Excelência em Gestão e Tecnologia; 2011; Rezende, RJ, Brasil. Resende: AEDB; 2011.

Oksanen J, Blanchet FG, Kindt R, Legendre P, Minchin PR et al. Community Ecology Package [online]. 2011 [citado em 2015 mar 30]. Disponível em: http://cran.r-project. org/web/packages/vegan/vegan.pdf

Rodrigues AC, Moreira MA. Análise dos reflexos sociais da aplicação dos royalties da mineração em Minas Gerais. In: Anais do X Simpósio de Excelência em Gestão e Tecnologia; 2013; Rezende, RJ, Brasil. Resende: AEDB; 2013.

Seabra AL, Khosrovyan A, Valls TAD, Polette M. Management of pre-salt oil royalties: wealth or poverty for Brazilian coastal zones as a result? Resources Policy 2015; 45: 1-8. http://dx.doi.org/10.1016/j.resourpol.2015.03.006.

Silva MAR. Royalties da mineração: instrumento de promoção do desenvolvimento sustentável de regiões mineradoras na Amazônia Oriental? Cadernos do IG/ UNICAMP 2000; 8(1-2): 3-21.

Thomé R. A função socioambiental da CFEM: compensação financeira por exploração de recursos minerais. Lisboa: DomTotal; 2009. [citado em 2015 mar 30]. Disponível em: http://www.domtotal.com/direito/uploads/pdf/59a a59814e06cb5bac839bd7d06aea9f.pdf

Varian HR. Intermediate microeconomics: a modern approach. 8. ed. New York: Norton e Company; 2010. 